\title{
Metastatic Adenocarcinoma: A Masquerade of Ring Enhancing Lesion of Brain
}

\author{
10.5005/jp-journals-10028-1331 \\ CPC Editor \\ Pathology Consultant \\ Clinical Discussant \\ Radiology Discussant \\ Senior resident \\ Clinician in charge \\ CPC Chairperson \\ Date of CPC \\ Date Of Admission \\ DOA of autopsy \\ CR number
}

\section{Case Description}

A 40-year-old lady presented with fever for the 1-month duration, skin rash for 7 days, and seizures with altered sensorium of 3 days duration. Seven months before the present illness, she was admitted in IGMC Shimla with complaints of seizures and fever for a short duration (8 days). Based on neuroimaging, a diagnosis of neurocysticercosis (NCC) was considered and the patient was started on antiepileptic drugs (sodium valproate (max $600 \mathrm{mg}$ ), albendazole, steroids ( $\max 40 \mathrm{mg}$ ), tapering doses, and stopped over 1 month), doxycycline, and cefoxime. Despite treatment, the patient kept having occasional seizures, headache, loss of weight, and appetite. The patient had stopped all the medications and visited PGI neurology out patient department (OPD) 1 month before the current admission where she was started on carbamazepine in view of partial epilepsy.

\section{EXAMINATION}

At admission, the lady was afebrile, having a pulse rate of 100 per minute, a blood pressure of $104 / 70$ mm of mercury, and a respiratory rate of 20 per minute. She had a maculopapular skin rash involving the whole body and oral mucosa. She was conscious but drowsy. Fundus examination revealed papilledema in both eyes. There was neck rigidity with a positive Kernig's sign. There was a normal pupillary reaction and there was flexor plantar response.

\section{InVESTIGATIONS}

Cerebrospinal fluid (CSF) analysis (13/4/15): total leucocyte count/differential leucocyte count (TLC/DLC): 10 cells/disfigured morphology

CSF analysis: protein/sugar: 66/54 mg/dL

Electrocardiogram (ECG): within normal limits

Urine routine examination (R/E): normal (September 2014); 5-7 cells, trace proteins (2/4/15)

HIV: negative

Hepatitis surface antigen ( $\mathrm{HBsAg}$ ): negative

Serum cholesterol: $192 \mathrm{mg} / \mathrm{dL}$

Triglyceride: $487 \mathrm{mg} / \mathrm{dL}$

Compression ultrasound B/L lower limbs—normal \begin{tabular}{l}
\hline 1,2,5 Department of Histopathology, Postgraduate Institute of Medical \\
Education and Research, Chandigarh, India \\
${ }^{3}$ Department of Neurology, Postgraduate Institute of Medical \\
Education and Research, Chandigarh, India \\
${ }^{4}$ Department of Radiology, Postgraduate Institute of Medical \\
Education and Research, Chandigarh, India \\
${ }^{6,7}$ Department of Medicine, Postgraduate Institute of Medical \\
Education and Research, Chandigarh, India
\end{tabular} Corresponding Author: Ritambhra Nada, Department of Histopathology, Postgraduate Institute of Medical Education and Research, Chandigarh, India, Phone: +91 7087008143, e-mail: ritamduseja@yahoo.com

How to cite this article: Nada R, Bal A, et al. Metastatic Adenocarcinoma: A Masquerade of Ring Enhancing Lesion of Brain. J Postgrad Med Edu Res 2019;53(3):133-141.

Source of support: Nil

Conflict of interest: None

ChestX-ray: normal(September2014), bilateral perihilar(12/4/15), consolidation (12/4/15), diffuse perihilar infiltrates? Pulmonary edema (17/4/15) Rest of investigations (refer to Tables 1 to 3 )

\section{Course and Management}

This 40-year-old lady was admitted to emergency services with a history of fever (1-month duration), seizures, and skin rash. In view of the previous history of NCC, possibilities of NCC with druginduced rash vs meningitis (? meningococcal) were considered. Neuroimaging (non contrast computarized tomography/contrast enhancing computerized tomography (NCCT/CECT) head) was suggestive of multiple ring-enhancing lesions. The patient was started on leveteracetam, decongestant therapy (mannitol and steroids (dexamethasone on 12/4/15, inj methyl prednisolone on 13/4/15, 14/4/15, and 16/4/15)), and antibiotics (Cloxacillin and Piperacillin/azobactum).

As per record, skin rash improved (>75\%) and methyl prednisolone was stopped on $16 / 4 / 15$ and the patient was started on oral prednisolone of $20 \mathrm{mg}$. The general condition of the patient worsened on 16/4/15 when she developed respiratory distress and

(O) The Author(s). 20190pen Access This article is distributed under the terms of the Creative Commons Attribution 4.0 International License (https://creativecommons. org/licenses/by-nc/4.0/), which permits unrestricted use, distribution, and non-commercial reproduction in any medium, provided you give appropriate credit to the original author(s) and the source, provide a link to the Creative Commons license, and indicate if changes were made. The Creative Commons Public Domain Dedication waiver (http://creativecommons.org/publicdomain/zero/1.0/) applies to the data made available in this article, unless otherwise stated. 
Table 1: Hemogram

\begin{tabular}{llllll}
\hline Date & September 2014 & $2 / 4 / 15$ & $12 / 4 / 15$ & $13 / 4 / 15$ & $14 / 4 / 15$ \\
\hline $\mathrm{Hb}(\mathrm{g} / \mathrm{dL})$ & 13.6 & 12.5 & 11.5 & 13.6 & 11.1 \\
$\mathrm{Plt}\left(\times 10^{3}\right)($ per $\mu \mathrm{L})$ & & 179 & 252 & 283 & 247 \\
$\mathrm{TLC}(\operatorname{per} \mu \mathrm{L})$ & 6,040 & 7,700 & 14,800 & $80 / 700$ & 9,900 \\
$\mathrm{DLC}(\mathrm{N} / \mathrm{L} / \mathrm{M} / \mathrm{E})$ & - & $77 / 20 / 2 / 1$ & - & - & - \\
$\mathrm{ESR}(\mathrm{mm}$ fall fistst & 25 & 18 & - & & 18 \\
hour & & & &
\end{tabular}

$\mathrm{Hb}$, hemoglobin; TLC, total leukocyte count; DLC, differential leukocyte count; Plts, platelets; ESR, erythrocyte sedimentation rate

Table 2: Biochemistry

\begin{tabular}{lllll}
\hline Date & September 2014 & September 2014 & $13 / 4 / 15$ & $16 / 4 / 15$ \\
\hline RBS (mg/dL) & 93 & - & - & - \\
$\mathrm{Na}^{+}(\mathrm{mmol} / \mathrm{L})$ & 140 & 134 & 136 & 128 \\
$\mathrm{~K}^{+}(\mathrm{mmol} / \mathrm{L})$ & 4.2 & 3.9 & 4.0 & 3.8 \\
Urea $(\mathrm{mg} / \mathrm{dL})$ & 34 & 28 & 25 & 20 \\
Creatinine $(\mathrm{mg} / \mathrm{dL})$ & 1.1 & 0.9 & 0.64 & 0.4 \\
Bilirubin total (mg/dL) & 1.3 & - & 1.1 & - \\
Bilirubin conj (mg/dL) & 0.4 & - & - & - \\
AST $(\mathrm{IU} / \mathrm{mL})$ & 52 & - & $\mathbf{2 8 7}$ & - \\
ALT (IU/mL) & 35 & - & $\mathbf{1 8 3}$ & - \\
ALP (IU/mL) & 68 & - & $\mathbf{8 6 4}$ & - \\
Protein total (g/dL) & 9.1 & - & 5.1 & - \\
& & & & - \\
Albumin (g/dL) & 4.5 & - & 2.7 & - \\
Ca++ (mg/dL) & 10 & - & $7.8(8.84)$ & - \\
CK-NAC (U/L) & - & - & 46 & - \\
PO4 (mg/dL) & 2.7 & - & - & - \\
Mg (meq./L) & - & - & 2.03 & - \\
\hline
\end{tabular}

RBS, random blood sugar; AST, aspartate transaminase; ALT, alanine transaminase; ALP, alkaline phosphatase; $\mathrm{Na}$, sodium; $\mathrm{K}$, potassium; $\mathrm{CK}$, creatine kinase; $\mathrm{Mg}$, magnesium; $\mathrm{Po}_{4}$, phosphate

Table 3: Arterial blood gas analysis

\begin{tabular}{lc}
\hline Date & $16 / 4$ \\
\hline $\mathrm{pH}$ & 7.536 \\
$\mathrm{pO}_{2}$ & 33.4 \\
$\mathrm{pCO}_{2}$ & 35.9 \\
$\mathrm{HCO}_{3}$ & 29.7 \\
$\mathrm{SpO}_{2} \%$ & 74.5 \\
\hline
\end{tabular}

hypotension. She sustained shock and cardiac arrest on 17/4/15 from which she could not be revived and was declared dead at 8:15 am.

\section{Dr Vivek Gupta:}

MRI brain (September 2014): Shows multiple areas of hyperintensities on T2-weighted imaging with moderate edema around it. Possibility of NCC or infective etiology is considered.

NCCT and CECT (April 2015): shows multiple ring lesions with enhancements seen in frontal lobe, temporal lobe, and occipital lobe in the gray-white matter junction which was hyperintense on non-contrast $\mathrm{CT}$ suggesting these to be hemorrhagic. These lesions were both in the supra- and infratentorial locations (Fig. 1).

Possibilities considered are metastasis, fungal infections, and remotely opportunistic infections.

CXR shows perihilar consolidation on the left lobe of the lung which could be either infective or because of aspiration.

\section{Case Analysis}

In a 40-year-old immunocompetent lady presenting with recent onset febrile illness, focal seizures with secondary generalization, and imaging suggestive of multiple ring-enhancing lesions, the first possibility considered would be infective granulomas such as NCC., Though the patient had been started on steroids, antiepileptics, and albendazole, she did not respond in the due course. She continued to have a headache and recurrent seizures with weight loss and loss of appetite. After a duration of 1 week of change of antiepileptic drug to carbamazepine, she developed with fever, skin eruptions, and seizures with altered mental status. These were probably due to the new drug added and responded to steroids. The altered mental status was due to raised intracranial pressure (ICP).

Differentials of hemorrhagic ring-enhancing lesions would be a fungal infection, nocardiosis, toxoplasmosis, metastasis, and tuberculomas (Table 4). Based on the rapid progression of the disease and the subsequent neuroimaging, initial diagnosis of NCC would be less likely. ${ }^{3}$ In imaging, there was less leptomeningeal enhancement with no basal or cisternal enhancement that makes the possibility of central nervous system (CNS) tuberculosis less likely. This is corroborated by normal sugar with a mild increase in protein in the CSF analysis. ${ }^{4}$ Toxoplasmosis is a stronger contender among the radiological differential diagnosis; however, the patient was negative for retrovirus and the lack of basal ganglia lesions makes toxoplasmosis less likely. ${ }^{5}$ Cerebral nocardiosis in the absence of lung lesions is also odd. The progression of the disease both clinically and radiologically points toward a metastasis.

Cerebral metastasis can be the initial manifestation of underlying malignancy in many cases. The three most common primary sources are lung carcinoma (40-50\%), breast carcinoma 

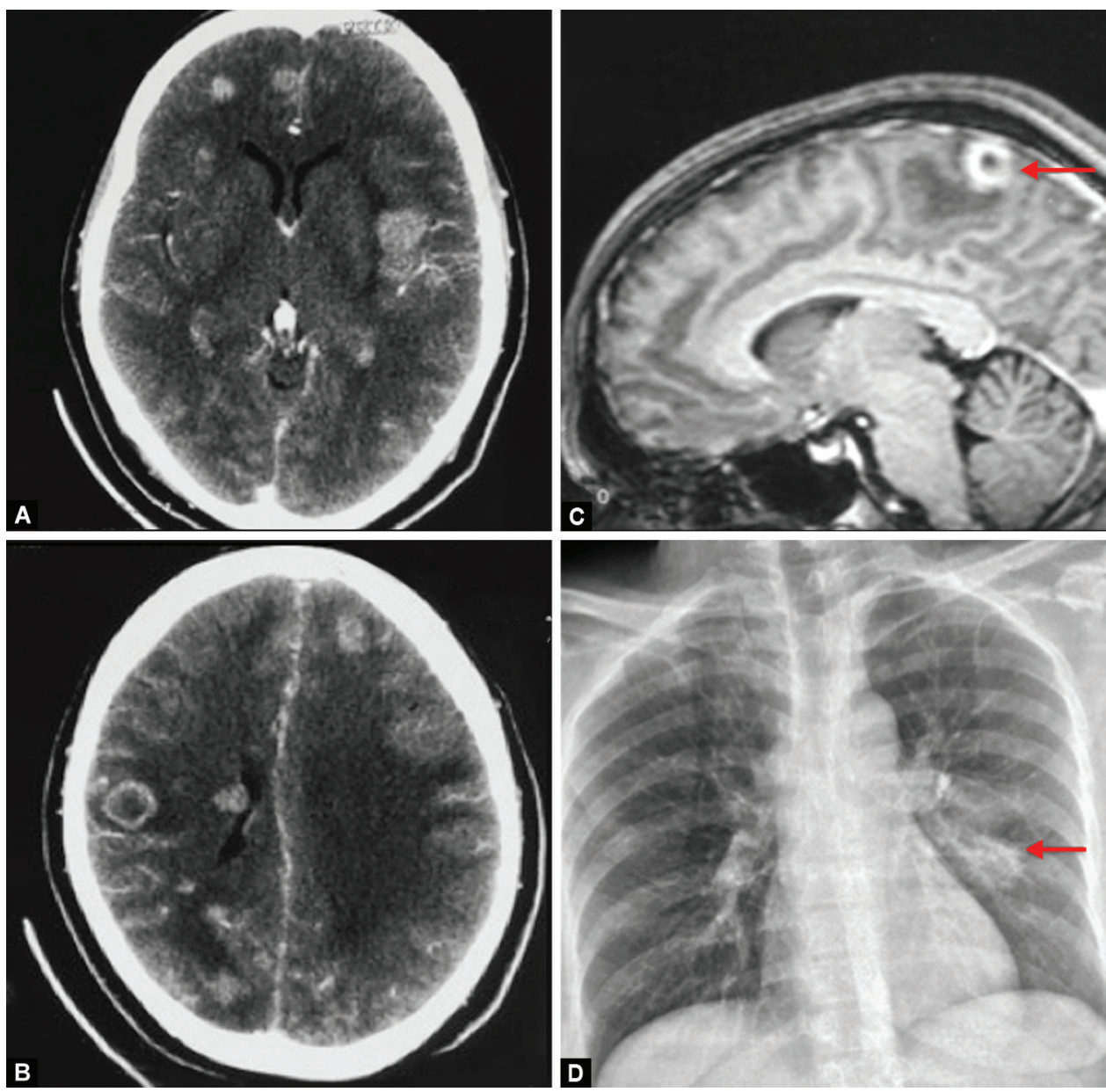

Figs $1 \mathrm{~A}$ to $\mathrm{D}$ : (A and $\mathrm{B})$ Contrast-enhanced $\mathrm{CT}$ images showing multiple ring enhancing lesions predominantly in the gray-white matter junction in the frontal, parietal, temporal, and occipital lobe; (C) Contrast-enhanced MRI sagittal plane shows a similar ring enhancing lesion in the parietal region; (D) Chest X-ray shows a perihilar shadow (arrow) in the left lower lobe retrospectively considering as the lung carcinoma

Table 4: Multiple ring enhancing lesions of the brain

\begin{tabular}{|c|c|c|c|c|}
\hline Bacterial & Fungal & Parasitic & Neoplastic & Inflammatory \\
\hline Pyogenic abscess & Nocardiosis & Cysticercosis & Metastasis & Multiple sclerosis \\
\hline Tuberculoma & Histoplasmosis & Toxoplasmosis & Primary brain tumor & $\begin{array}{l}\text { Acute disseminated } \\
\text { encephalomyelitis (ADEM) }\end{array}$ \\
\hline Mycobacterium avium & Aspergillosis & Amebic abscess & Primary CNS lymphoma & Sarcoidosis \\
\hline Syphilis & Cryptococcosis & Echinococcosis & & Neuro-Behcet's \\
\hline \multirow[t]{3}{*}{ Listeriosis } & Actinomycosis & Sparganosis & & Whipple's disease \\
\hline & Coccidioidomycosis & Chagas' disease & & SLE \\
\hline & Mucormycosis & & & \\
\hline
\end{tabular}

(15-25\%), and melanoma (5-20\%). ${ }^{6}$ Twenty percent of cases may not have the primary site of tumor identified during the lifetime.

Transaminitis in this patient could have been caused by carbamazepine intake or by tumor infiltration.

Terminal events could be because of aspiration that induces aspiration pneumonitis (ARDS) or a thromboembolism due to a procoagulant state caused by malignancy.

\section{Final Clinical Diagnosis}

- Multiple ring-enhancing lesions in the brain

- Metastasis (Primary;? Breast?? Ovarian)

- Infectious (? Tuberculoma? Toxoplasmosis? Nocardia)
- Carbamazepine-induced drug rash

- ARDS.

\section{Clinical Discussion}

Prof Subash Varma. Comments from the treating unit.

Prof Ashish Bhalla: The case has been very well discussed. During the time when the patient was admitted with us, there were two things we were trying to control. One was raised ICP and the second was the skin rashes, which we thought could be because of the antiepileptic drug she was taking. That's why pulse methyl prednisolone was given to control the skin rash and antibiotics were given to cover the lung infiltrates and the secondary Staphylococcus 

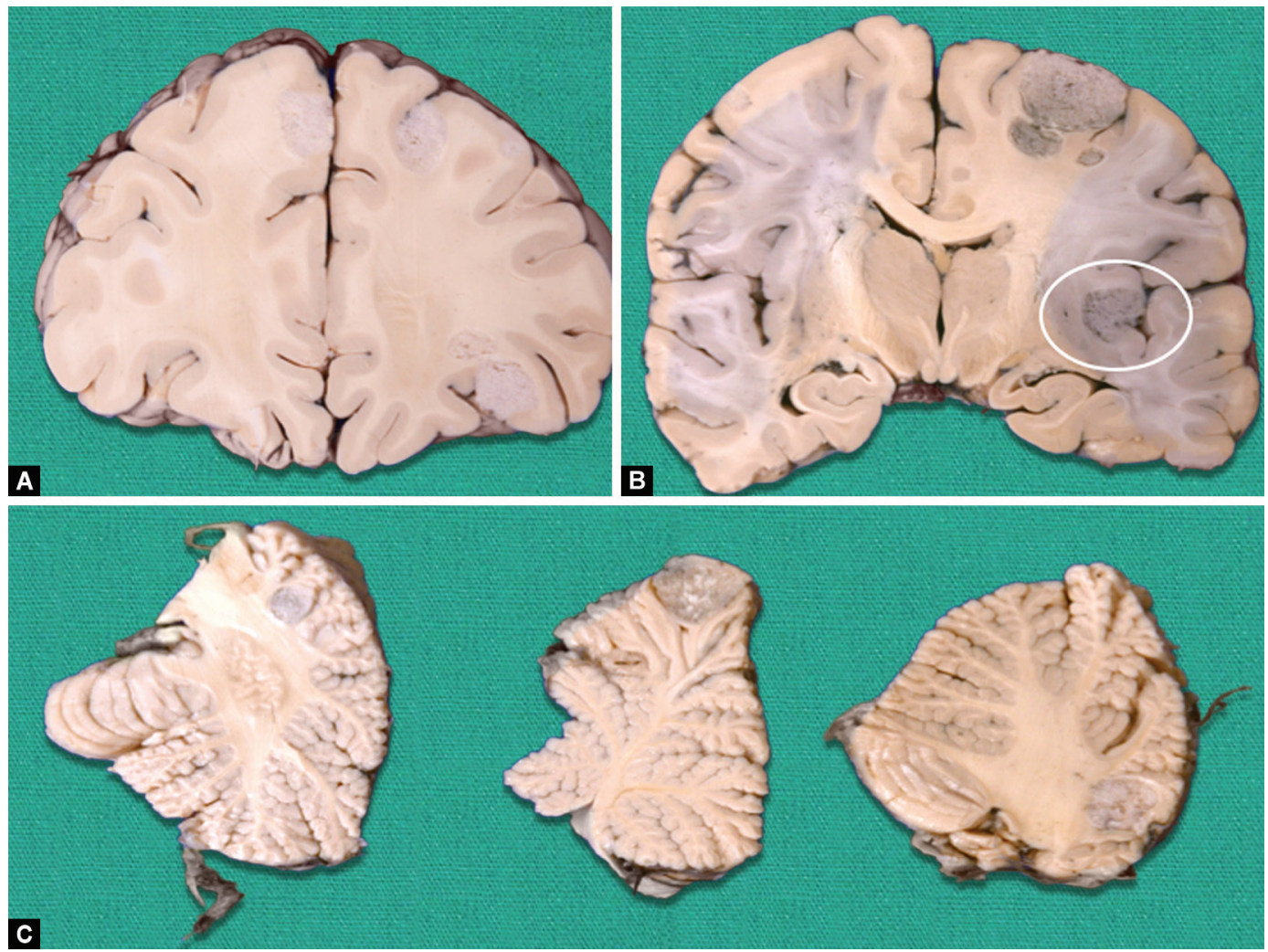

Figs $2 \mathrm{~A}$ to $\mathrm{C}$ : (A) Gross photograph of the anterior slices of the brain shows multiple grayish nodular lesions located in the gray-white matter junction in the frontal and temporal lobes; (B) Coronal slices at the level of basal ganglia shows similar metastatic deposits in the insular cortex (circled); (C) Metastatic deposits are noted in the cerebellum

infection of the skin lesions. The terminal event could be because of flaring up of the lung lesion and may not be attributed to the ARDS since the patient received methylprednisolone at a higher dose. One can have flaring up of the infection. There is nothing in radiology to suggest a primary lung lesion including malignancy. I do not think gynecological examination was done because it was not in the primary consideration; that's what we should have looked for. The patient needed more investigation in that front. In the emergency, the aim was to control the skin lesions and the raised ICP along with covering for the infection.

Prof Vanita Suri: In this patient, though the chest X-ray was normal, one must always think about choriocarcinoma, especially in women. A urine pregnancy test should always be done in conditions such as brain lesion, seizures, or malignancy.

Dr Gaurav Prakash: Classical brain metastasis presenting as a ring-enhancing lesion is not a very common finding. Metastatic renal cell carcinoma and melanoma are the two most common primaries. Unfortunately, we do not have any information regarding skin examination apart from rash. Breast and gynecological examinations are mandatory during the evaluation of space occupying lesions (SOLs) in the brain. By the speed of deterioration and demise, I believe this a case of malignancy.

Prof Vivek Lal: There are two important components in this case: the clinical and the radiological profile. If we look at the clinical profile, this patient has a very rapid downhill course. Till September, she was well, then she has a brief illness. She has brief seizures and fever but she continues to lose weight and appetite. But when she comes here in the month of April in a conscious drowsy state, within 5 days, it is all over. So near the end, the disease gained momentum. Note one thing that she has a CSF that shows only 10 cells, normal sugar, and marginally elevated proteins that exclude an infective etiology like fungal. But then, this patient also had neck rigidity and positive kernig' sign, which implied that she was coning because of raised ICP and SOL. If the first MRI was given to me, I would make a diagnosis of NCC. But the subsequent CT scan in this admission leaves no scope but to deny the original MRI findings. The CT scan and these CSF findings would make me consider a noninfective etiology like a metastasis from an unknown site. A choriocarcinoma, in view of hemorrhage, would certainly be a possibility followed by renal cell carcinoma.

Prof Manoj: Multiple lesions, with significantly raised ICP and areas of hemorrhage, a possibility of metastasis is more likely.

Dr Varun: One thing, which is not explained by metastasis, is fever. During both the time of presentation, in IGMC and PGI, the patient had a fever with hypergammaglobulinemia. In view of this, could it still be tuberculosis.

Dr Aastha Takkar: The low-grade fever was already going upon and that is why metastasis was considered. During the next presentation, the fever can be attributed to the carbamazepineinduced drug rash.

Prof Modi: With the presence of hemorrhagic lesions, tuberculosis is highly unlikely. We have our own serial MRI data in CNS tuberculoma in which we have not seen a hemorrhagic lesion. We should have doubted the diagnosis of cysticercosis when there were only a few cysticerci lesions. If SWI films would have been given, we would have thought about hemorrhagic lesions.

Prof Subhash Varma: You think hemorrhagic lesions were present in the first MR?

Prof Vivek Gupta: First MRI was a conventional MRI. It does not show any evidence of hemorrhage. There was no T2 hypointensity. 


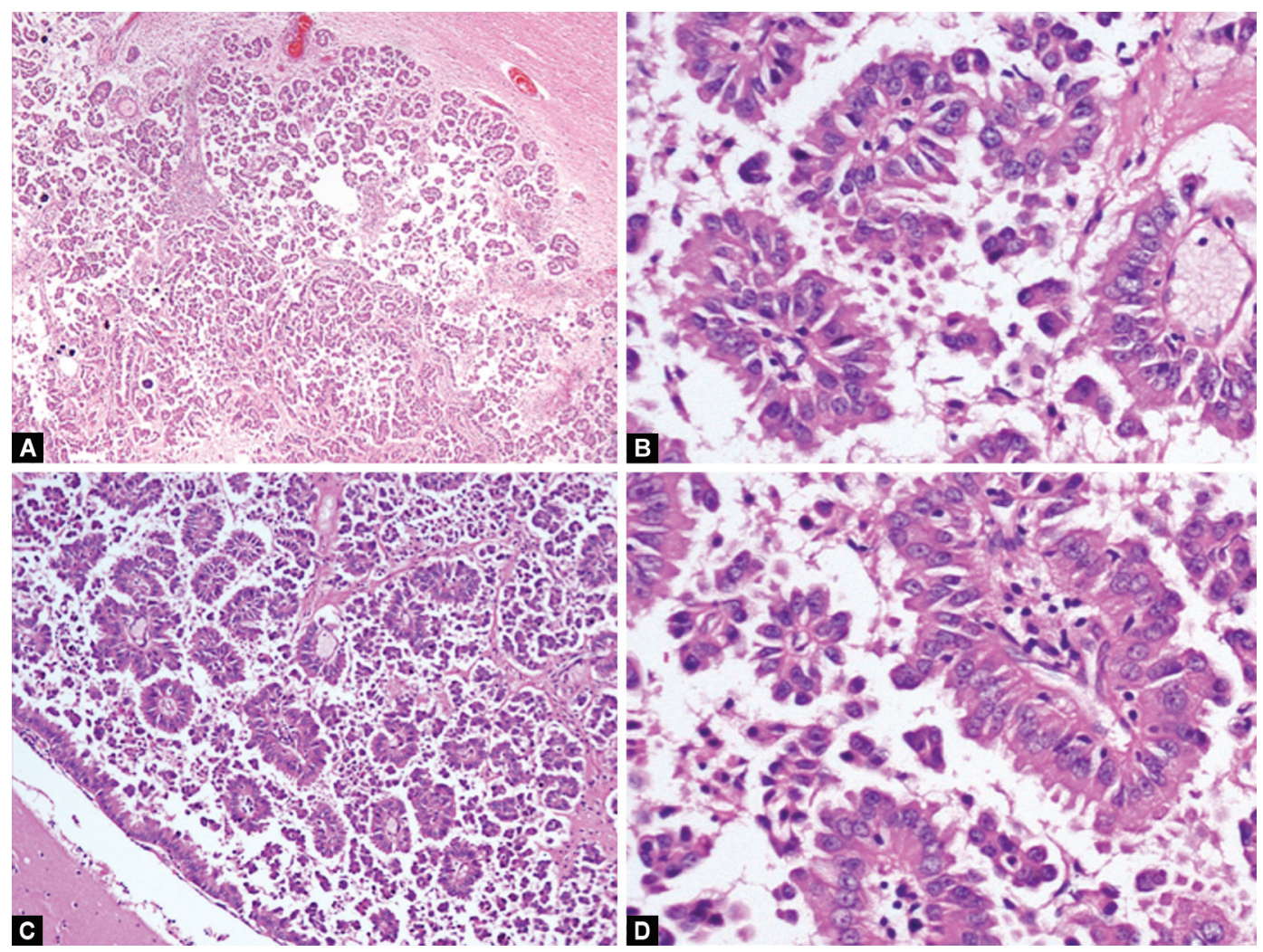

Figs 3A to D: (A) Microscopy from the brain lesion showed a metastatic adenocarcinoma arranged in the form of papillary and micropapillary pattern (hematoxylin- and eosin-stained section, 40× magnification); (B) Higher magnification of the tumor arrangement-micropapillary pattern (hematoxylin- and eosin-stained section, 400× magnification); (C) The left lower corner shows the white matter of the brain with the tumor present in gray-white junction (hematoxylin- and eosin-stained section, 100× magnification); (D) Micropapillary tumor pattern showing reverse polarity of the tumor cells (hematoxylin- and eosin-stained section, 400x magnification)

Tuberculoma would have been considered if there was T2 hypointensity.

Prof Paramjit: I think we are overemphasizing on hemorrhage. There is something called visual paradox in radiology. Artefactually increased intensity over a dark background is the paradox.

Prof Subhash Varma: In today's case, we have a young woman with multiple ring-enhancing lesions that progressed while on treatment. Initial impression was NCC. Infectious etiology on treatment for 6 months duration is lower down the line. We are considering metastasis today. The liver lesion could be because of carbamazepine intake rather than metastasis.

The primary site is the question now. Many possibilities of the primary site were discussed.

I now invite Dr Amanjit Bal for presenting the pathology protocol.

\section{Pathology Findings}

A complete autopsy was performed. The prosectors noted the deceased to be of moderate build. The pleural, pericardial, and peritoneal cavities were within normal limits.

The brain weighed $1,955 \mathrm{~g}$. There was cerebral edema noted in the form flattening of gyri and close opposition of sulci. Right cerebellar tonsillar herniation was noted. There was a small depression in the parietal region. On slicing, multiple well-defined nodular lesions were noted in bilateral superior frontal gyri, parietal cortex, middle frontal, basal, basifrontal, occipital lobe, and cerebellar hemispheres (Fig. 2). These lesions were located in the gray-white junction varied in size from $3 \mathrm{~mm}$ to $2 \mathrm{~cm}$ with focal cystic degeneration. Microscopic examination of these nodules showed metastatic adenocarcinoma with papillary and micropapillary histologic patterns (Fig. 3). The tumor cells were immunopositive for CK7, Napsin, and TTF1, while they are negative for CK20. EMA highlighted the reverse polarity in the micropapillary pattern (Fig. 4).

A micropapillary pattern of lung adenocarcinoma in brain metastasis is associated with lesser overall survival. ${ }^{7}$

Lungs: Both the lungs weighed 1,350 g. Both of them were heavy and sub-crepitent. The pleura was shiny. The cut surface of the left lung showed a firm grayish-white lesion with mucoid surface in the lower lobe measuring $5 \times 4 \mathrm{~cm}$. It was located close to the main bronchus. The pleural surface over this lesion was dull. Another tiny nodule of $0.5 \mathrm{~cm}$ was seen near the larger nodule. The hilar and carinal lymph nodes were enlarged measuring $0.5-1.5 \mathrm{~cm}$. No thrombus was seen in the major pulmonary vessels. A microscopic examination from the firm gray-white lesion of the left lung showed a tumor arranged in an acinar, papillary, and micropapillary pattern. The tumor cells had round to oval nuclei, vesicular chromatin, and prominent nucleoli. There were foci of intra-parenchymal spread (same side) and lymphatic tumor emboli (Fig. 5).

\section{Molecular Analysis}

The tumor was negative for known epidermal growth factor receptor (EGFR) mutations in Exons 18, 19, 20, and 21 by the EGFR mutation analysis kit by real-time polymerase chain reaction (PCR). Tumor cells were positive ALK-1 (D5F3 clone, Cell Signaling) which was later confirmed by the ALK break apart FISH (Fig. 5). 


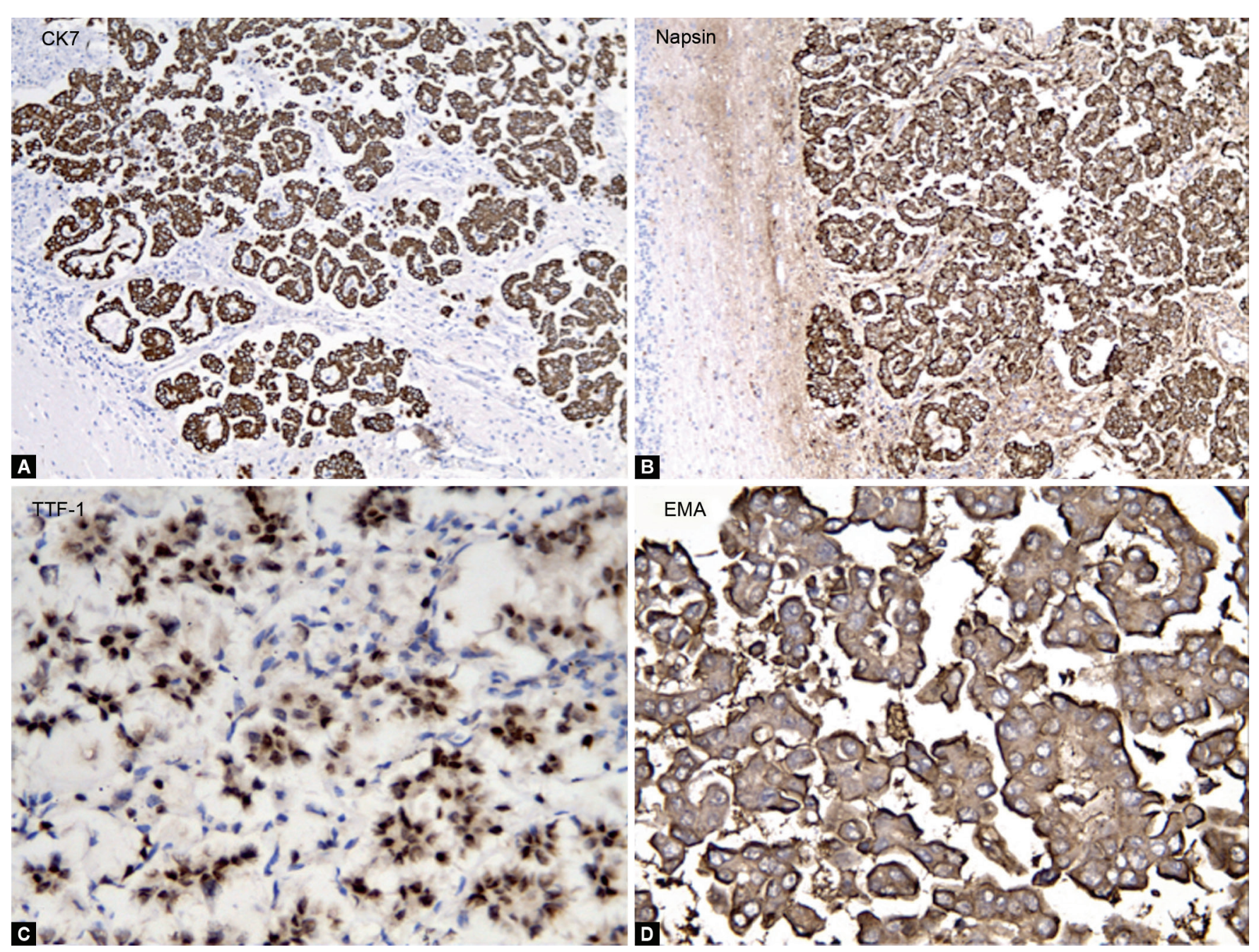

Figs 4A to D: Immunohistochemistry panel of tumor cells from A to D; (A) The tumor cells are diffusely positive for CK-7 (membranous pattern)

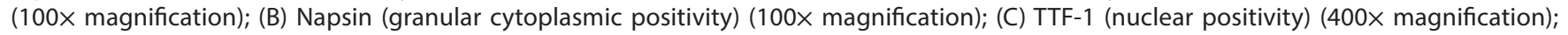
(D) EMA stain shows preferential staining of the periphery of the micropapillae (400× magnification)

ALK-positive lung adenocarcinoma represents approximately $4-5 \%$ of all NSCLC. The salient features are the following:

- Patients are relatively younger and never or light $(<10$ packyears) smokers

- In up to $46 \%$ of ALK+ NSCLC-, the central nervous system is the first site of progression

- Respond well to small molecule ALK inhibitors such as crizotinib

The incidence of synchronous brain metastases in newly diagnosed stage IV ALK-positive NSCLC ranges from 20 to $30 \%$. Cystic brain metastasis has been well observed in ALK-positive lung carcinomas. ${ }^{8}$

In addition, there was evidence of bronchopneumonia, pulmonary edema, and a few organized thrombi in pulmonary vessels. Incidental chondroid hamartoma was also noted (Fig. 5).

Uterus and ovaries: Section from the endometrium shows the proliferative phase. Ovaries grossly show follicular cysts. Microscopic examination of both showed a similar metastatic adenocarcinoma (Fig. 6).

Skin: Section from the epidermis showed vacuolation of the basal layer and lymphocytic exocytosis. Dermis showed perivascular lympho-histiocytic infiltrate admixed with a few eosinophils. The lymphocytes were predominantly CD8 positive with occasional CD4-positive cells.

The heart weighed $284 \mathrm{~g}$. The pericardial surface was unremarkable. There was a mild dilatation of the right atrial and the right ventricular cavity. The ventricular wall thicknesses were within normal limits. Valves were within normal limits and no vegetations were seen. There was diffuse discoloration noted in the left ventricular wall. Microscopy of the discolored area showed extensive interstitial edema, focal myocardial necrosis, and inflammatory infiltrate comprising of lymphocytes (CD8 predominant) and macrophages (CD68 positive) indicating myocarditis (Fig. 7).

The skeletal muscle showed focal similar to lymphohistiocytic inflammatory infiltrate.

The liver weighed 1,310 g. Grossly, the capsular surface was normal with no wrinkling or nodularity. The cut surface was mottled. Biliary tree, portal, and hepatic veins were normal. Microscopy showed preserved a lobular architecture with centrizonal areas of hemorrhagic necrosis noted with reticulin collapse. Portal tracts and hepatic parenchyma showed ill-formed epithelioid cell granulomas 

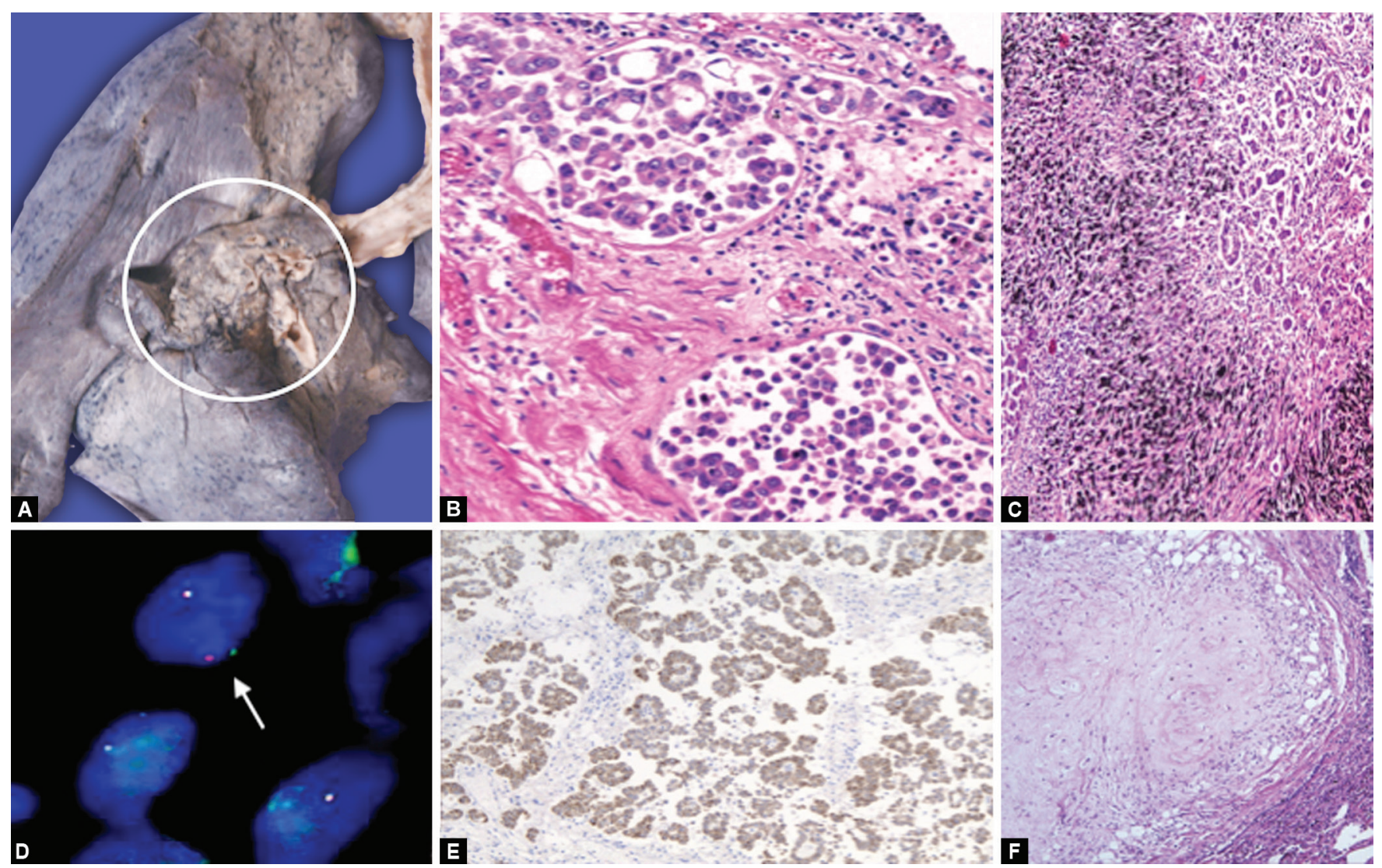

Figs 5A to F: (A) Grossly the left lung showed a gray-white mass lesion near the hilum; (B) Microscopic section from the mass shows similar adenocarcinoma (hematoxylin- and eosin-stained section, 200× magnification); (C) Metastatic adenocarcinoma in the hilar lymph nodes (hematoxylin- and eosin-stained section, 200× magnification); (D) ALK breakapart FISH shows split signals, one green and one red; (E) ALK D5F3 clone shows diffuse positivity (200x magnification); (F) Incidentally detected chondroid hamartoma (hematoxylin- and eosin-stained section, $400 \times$ magnification)

with occasional giant cells and eosinophils. The stain of acid-fast bacilli is negative (Fig. 7).

The bone marrow examination revealed an adequate representation of all marrow elements. A few non-caseating epithelioid cell granulomas with occasional giant cell noted. There was an increase in eosinophilic precursors.

Features like myocarditis, myositis, vacuolar interface dermatitis (rash), and non-caseating granulomas in the liver point toward the carbamazepine-induced drug reaction with eosinophilia and systemic symptoms (DRESS) syndrome (Fig. 7).

Features of the DRESS syndrome (HLA typing: positive for HLA$A^{*} 31: 01$, Negative for HLA-B*15:02) are the following:

- The DRESS syndrome presents as an extensive mucocutaneous rash, fever, lymphadenopathy, hepatitis, and hematologic abnormalities with eosinophilia.

- Liver involvement is the most common visceral manifestation (50-60\%).

- Myocarditis may develop at the start or up to 40 days after emergence of the drug reaction. ${ }^{9}$

- The two common risk variants described for carbamazepineinduced hypersensitivity reactions are HLA-B*15:02 and HLAA*31:01. ${ }^{10}$

Spleen weighed $180 \mathrm{~g}$. The cut surface was congested. Microscopy showed numerous non-caseating epithelioid cell granulomas with giant cells, mostly centered on blood vessels. The stain for acid fast bacilus (AFB) was negative. However, PCR for Mycobacterium tuberculosis was positive (Fig. 6). The granulomas in the spleen were more compact compared to the ones in the liver.

Kidneys: Both weighed $240 \mathrm{~g}$. The outer surface was unremarkable. The cut surface showed distinct corticomedullary junction with medullary congestion. Microscopic examination showed mild vacuolar degeneration in the tubules. Glomeruli, interstitium, and blood vessels show no significant pathology (Fig. 6).

Gross and microscopic examination from the gall bladder, pancreas, esophagus, stomach, small and large intestine, and adrenal glands did not show any significant pathological findings.

\section{Final Autopsy Diagnosis}

A 40-year-old female on carbamazepine for seizures:

- Adenocarcinoma lung, papillary predominant pattern (ALK positive)

- Metastasis to the brain and the ovaries

- Features of the DRESS syndrome (HLA-A*31:01 associated)

- Skin involvement, granulomatous hepatitis, increased eosinophilic precursors in bone marrow, and myocarditis

- Predominant splenic tuberculosis 

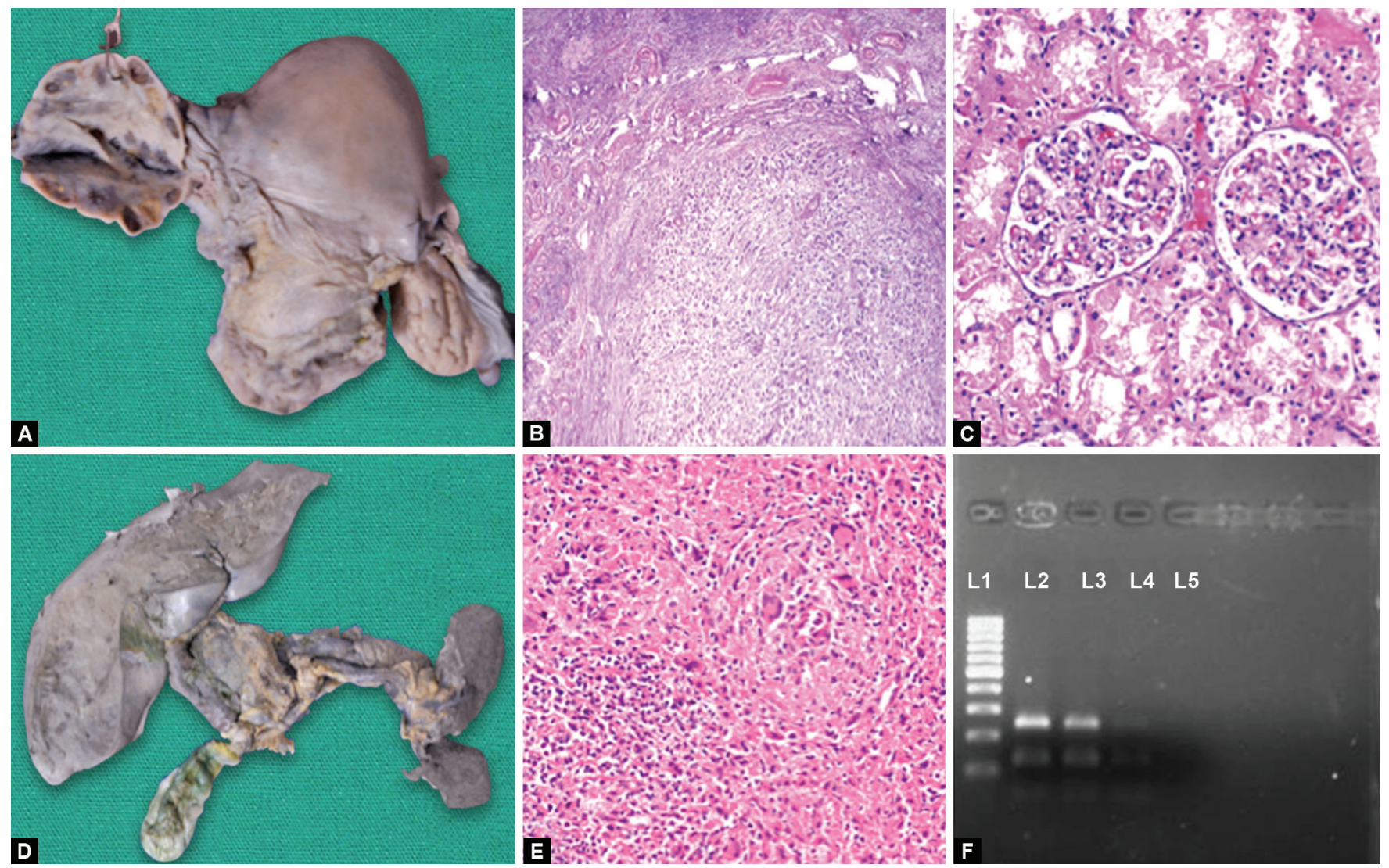

Figs 6A to F: (A) Gross photograph of the uterus and the ovary. The cut surface of the ovary shows multiple cystic follicles; (B) Microscopic section from ovary shows metastatic adenocarcinoma (hematoxylin- and eosin-stained section, 100x magnification); (C) Representative section from the kidney shows normal glomeruli and tubules; (D) Organ complex showing the liver, the spleen, and the pancreas with normal outer and cut surfaces; (E) Section from the spleen shows non-caseating epithelioid cell granuloma and Langhan's giant cells (hematoxylin- and eosin-stained section, 400× magnification); (F) PCR for Mycobacterium tuberculosis is positive (L1—100 bpmm, L2 - positive control, L3 and L4—DNA extracted from the spleen and LN tissue positive for M. tuberculosis, and L5-NC)

\section{Final Discussion}

Prof Subhash Varma: Thank you Dr Amanjit. A very good job of knitting the overall story. Both the clinical and pathological protocols are open for discussion.

Prof Ashish Bhalla: Nice presentation of the pathological protocol. We were right in two fronts. One is carbamazepineinduced drug reaction. There was no infection in the brain and we have to start trusting our CSF reports (normal sugar with low cell count). There are two lessons to be learned. Any lesion in the lung should not be ignored and aspiration does not occur in the left lobe. The terminal events have occurred because of the flare-up of infection caused by a high dose of steroids leading to respiratory involvement.

The question to the radiologist: Can we retrospectively think of metastasis in the first MRI?

Prof Vivek Gupta: Even retrospectively, it is difficult to consider metastasis in a patient presenting with short duration of seizures, fever with ring, and disc lesion without edema.

Regarding the chest X-ray lesion: A single opacity is seen only one time, so it is difficult to call it as malignancy.

Prof Subhash Varma: I would agree with you. However, it is also known that many of the shadows appear larger on chest X-ray follow-up.

Prof Bishan Radotra: I would like to say that melanoma hardly metastasizes to the brain. We have seen the primary melanomas of the spinal cord and the brain. And, in our series of metastatic carcinomas in the brain, the lung is the most common cause of primary, especially in adult males. Breast metastasis is usually solitary whereas lung metastasis is multiple. Hemorrhagic lesions were not seen during brain cutting also. Mucin-secreting adenocarcinomas can perplex the radiologists. Tuberculomas with hemorrhage in its wall is also rare.

Prof Varinder Singh: There was splenic tuberculosis. But with regard to granulomas in the liver, why was it presented as carbamazepine-induced granulomas? Why not tuberculosis.

Prof Amanjit: Both compact and loose granulomas were present in the liver and the spleen. So it could be a combination of drug induced and tuberculosis.

Prof Subhash Varma: The granulomas in the liver, skin lesion, and inflammation in myocardium fits better with the DRESS syndrome and that is why it would have been considered.

Prof Behra: It is a learning experience for both neurologists and radiologists. Metastasis workup should have been done for this patient. Prof Sanjay Jain: Is magnetic resonance sonography (MRS) sensitive for disc lesions?

Prof Vivek Gupta: MRS is essential to characterize the lesion rather than picking up the lesion. Had the MRS been done and if choline would have been present, probably it could have picked up the metastasis. MRS is routinely done in our institute.

Prof Parampreet: It is important to educate the patient about rashes when drugs like carbamazepine are given. Especially, 


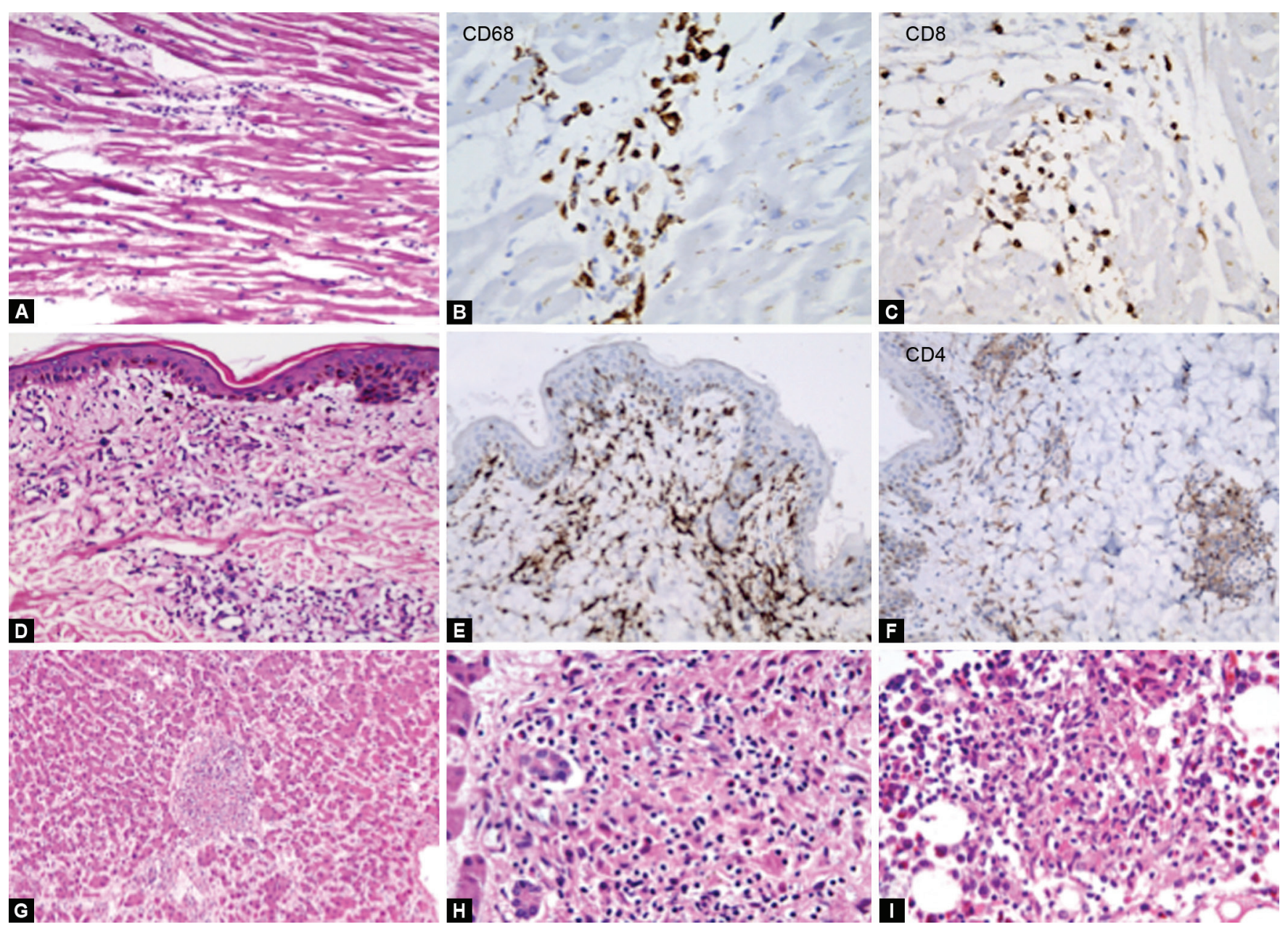

Figs 7A to I: Panel of photographs representing the DRESS syndrome; (A) Myocardium shows lymphohistiocytic infiltrate and edema with myocardial necrosis (hematoxylin- and eosin-stained section, 200× magnification); (B) The infiltrate is rich in CD68 highlighting the histiocytes $(400 \times)$; (C) The lymphocytes dominantly comprise of CD8+ population (400X); (D) Skin biopsy shows vacuolar degeneration, pigment incontinence, and perivascular lymphocytic infiltrate (hematoxylin- and eosin-stained section, 100x magnification); (E and F) The infiltrate is rich in CD8 than CD4 (200x magnification); (G) Liver histology shows portal ill-defined granulomas (hematoxylin- and eosin-stained section, 40× magnification); (H) Granulomas in the liver (hematoxylin- and eosin-stained section, 400× magnification); (I) Bone marrow shows trilineage hematopoiesis with occasional ill-defined granulomas (hematoxylin- and eosin-stained section, 400x magnification)

when it has a potential to reach up to the Steven Johnson syndrome.

Prof Arvind Rajwanshi: There are many lessons to be learned from this case. Repeated CSF examination should have been done and probably it would have picked up the metastasis.

Prof Subhash Varma: Thank you very much. Today's case has taught valuable lessons such as limitations in examination, limitations in investigations, and its interpretation. Last is the course of illness, which will provide a better clue. In today's case, it is the lack of response to a steroid that made one to reconsider the initial diagnosis and one patient can have more than one diagnosis.

\section{References}

1. Garg R, Sinha M. Multiple ring-enhancing lesions of the brain. J Postgrad Med 2010;56(4):307. DOI: 10.4103/0022-3859.70939.

2. Shetty G, Avabratha KS, et al. Ring-enhancing lesions in the brain: a diagnostic dilemma. Iran J child Neurol 2014;8(3):61-64.

3. Garcia HH, Nash TE, et al. Clinical symptoms, diagnosis, and treatment of neurocysticercosis. Lancet Neurol 2014 Dec;13(12):1202-1215. DOI: 10.1016/S1474-4422(14)70094-8.
4. Thwaites G. Neurological aspects of tropical disease: Tuberculous meningitis. J Neurol Neurosurg Psychiatry 2000 Mar 1;68(3):289-299. DOI: 10.1136/jnnp.68.3.289.

5. Lee GT, Antelo F, et al. Cerebral Toxoplasmosis. RadioGraphics 2009 Jul;29(4):1200-1205. DOI: 10.1148/rg.294085205.

6. Eichler AF, Loeffler JS. Multidisciplinary Management of Brain Metastases. Oncologist 2007 Jul 1;12(7):884-898. DOI: 10.1634/ theoncologist.12-7-884.

7. Casteillo F, Guy JB, et al. Pathologic Subtypes of Lung Adenocarcinoma Brain Metastasis Is a Strong Predictor of Survival After Resection. Am J Surg Pathol 2018 Dec;42(12):1701-1707. DOI: 10.1097/ PAS.0000000000001161.

8. Rusthoven CG, Doebele RC. Management of Brain Metastases in ALK -Positive Non-Small-Cell Lung Cancer. J Clin Oncol 2016 Aug 20;34(24):2814-2819. DOI: 10.1200/JCO.2016.67.2410.

9. E L omairi N, Abourazzak S, et al. Drug Reaction with Eosinophilia and Systemic Symptom (DRESS) induced by carbamazepine: a case report and literature review. Pan Afr Med J 2014;18:9.

10. Amstutz U, Shear NH, et al. Recommendations for HLA-B*15:02 and HLA-A*31:01 genetic testing to reduce the risk of carbamazepineinduced hypersensitivity reactions. Epilepsia 2014 Apr;55(4):496-506. DOI: 10.1111/epi.12564. 